\title{
Dialektmerkmale des Deutschen in den sprachbiographischen Interviews der bilingualen Oberschlesier
}

\author{
Felicja Księżyk (D)
}

Eingegangen: 1. Februar 2021 / Angenommen: 21. Mai 2021 / Online publiziert: 15. Februar 2022 (C) Der/die Autor(en) 2022

Zusammenfassung Der Artikel stützt sich auf sprachbiographische Interviews, die im Rahmen des deutsch-polnischen Projekts LangGener mit einheimischen Bewohnern von ehemals deutschen Gebieten im heutigen Polen durchgeführt wurden. Von den insgesamt 124 Aufnahmen werden 20 sprachbiographische Interviews mit Sprechern und Sprecherinnen in Oberschlesien einer Analyse im Hinblick auf die darin vorkommenden remanenten Merkmale der schlesischen deutschen Dialekte unterzogen. Auch wenn die schlesische Sprachlandschaft in der Forschung vor dem Hintergrund der Grenzverschiebungen nach dem Zweiten Weltkrieg mit dem damit einhergehenden Bevölkerungsaustausch nunmehr für einen historischen Untersuchungsgegenstand erachtet wird, so zeigt der Artikel, dass auch die aktuell im LangGener-Korpus gewonnenen sprachlichen Daten restdialektale Merkmale des Deutschen aufzeigen. Entgegen der oft vertretenen Ansicht, wonach sich das oberschlesische Deutsch insbesondere durch sprachkontaktinduzierte Eigentümlichkeiten vom Standarddeutsch abheben würde, wird bewiesen, dass sprachkontaktbedingte Veränderungen nicht immer das alleinige und größte Erklärungspotenzial für manche im oberschlesischen Deutsch der Interviewten auftretende Phänomene haben. Zahlreiche Besonderheiten lassen sich als Anzeichen von Dialektkontinuität einstufen.

Schlüsselwörter Varietäten des Deutschen · Oberschlesien · Zweisprachigkeit · Dialektmerkmale · Vom Aussterben bedrohte Varietäten

Felicja Księżyk ( $\square)$

Uniwersytet Opolski, Opole, Polen

E-Mail: ksiezykf@uni.opole.pl 


\title{
Dialect Features of German Language in the Language Biographies of Bilingual Upper Silesians
}

\begin{abstract}
The article is based on language biographies which come from the interviews conducted as part of the German-Polish project LangGener with native inhabitants of the former German territories of today's Poland. Out of 124 recordings, 20 linguistic biographical interviews with respondents from Upper Silesia were subjected to analysis where special attention was paid to the features of Silesian German dialects occurring in them.

Even though the Silesian linguistic landscape is now considered by researchers to be a historical object of studies due to the shifting of borders after World War II and the accompanying population exchange, the article shows that the linguistic material currently obtained in the LangGener corpus also reveals residual dialectal features of the Silesian German dialects. Contrary to the common view that Upper Silesian German differs from standard German primarily by features resulting from linguistic contact, it has been proved that changes resulting from linguistic contact do not always have the only or the greatest potential to explain some phenomena occurring in the Upper Silesian German of the surveyed informants. Numerous features can be classified as signs of dialectal continuity.
\end{abstract}

Keywords Varieties of German - Upper Silesia · Bilingualism · Dialect Features · Endangered Varieties

\section{Einführung 1}

Der vorliegende Artikel stützt sich auf Untersuchungsergebnisse, die im Rahmen des deutsch-polnischen Projekts LangGener (Generationsbedingte Differenzierung der Sprache: morphosyntaktische Veränderungen durch deutsch-polnischen Sprachkontakt in der Sprache zweisprachiger Personen) erzielt wurden. In den Jahren 2018-2019 führten die Projektmitarbeiter zahlreiche Feldforschungen mit Vertretern zweier Generationen durch:

1. Einer älteren Generation, die in Polen lebt, in den Gebieten, die bis $1945 \mathrm{zu}$ Deutschland gehörten. Diese Informanten sind vor 1945 und mehrheitlich in den 1930er-Jahren geboren.

2. Einer jüngeren Generation mittleren Alters, die in den 1950er- und 1960er-Jahren in Polen in denselben Gebieten geboren wurde und derzeit in Deutschland lebt. Sie sind in den 1970er-, 1980er- und 1990er-Jahren ausgewandert.

\footnotetext{
1 Der Artikel wurde im Rahmen des Forschungsprojekts »Generationsbedingte Differenzierung der Sprache: morphosyntaktische Veränderungen durch deutsch-polnischen Sprachkontakt in der Sprache zweisprachiger Personen « verfasst (im Folgenden als LangGener abgekürzt), finanziert aus Mitteln des Nationalen Wissenschaftszentrums (Narodowe Centrum Nauki/National Science Centre, Poland), Projektnr. 2016/23/G/HS2/04369, realisiert vom Institut für Slawistik der Polnischen Akademie der Wissenschaften.
} 
Bis Oktober 2019 konnten sprachbiographische Interviews mit 124 Informanten, jeweils zur Hälfte auf Polnisch, zur Hälfte auf Deutsch, durchgeführt werden. Die gewonnenen sprachlichen Daten sollen dazu verhelfen, mehrere Forschungsziele $\mathrm{zu}$ verfolgen. Angestrebt wird damit 1. die Erstellung eines Korpus der deutschpolnischen Zweisprachigkeit, 2. die Beschreibung des Sprachgebrauchs bei zwei Generationen von deutsch-polnischen Bilingualen sowie 3. die Entwicklung einer Typologie der morphosyntaktischen Musterreplikationen (sog. Pattern Replications nach Heine/Kuteva 2005, S. 40). Als ein Unterziel des zweiten hier genannten Hauptziels ist die Dokumentierung des in Polen noch heute gesprochenen Deutschen im Hinblick auf die dialektalen Merkmale zu betrachten. Im Folgenden sollen die remanenten dialektalen Merkmale, die von den Sprechern in Schlesien bei ihrem Deutschgebrauch noch heute realisiert werden, näher beleuchtet werden.

\section{Zu den Probanden}

Im Rahmen des Projekts LangGener wurden 20 sprachbiographische Interviews mit Vertretern der älteren Generation durchgeführt, die noch heute in Oberschlesien leben (mit 9 Männern und 11 Frauen). Es handelt sich dabei um 21,5 Stunden Aufnahmen auf Polnisch und 22,5 Stunden auf Deutsch.

Die Herkunftsorte der Interviewten liegen in der Woiwodschaft Oppeln und Schlesien und zwar in der Woiwodschaft Oppeln in den Kreisen: Kandrzin-Cosel (Kędzierzyn-Koźle), Neustadt (Prudnik), Krappitz (Krapkowice), Oppeln (Opole), Groß Strehlitz (Strzelce Opolskie) und in der Woiwodschaft Schlesien in dem Kreis Ratibor (Racibórz). Dass der Großteil der Informanten aus der Woiwodschaft Oppeln stammt, rührt daher, dass dort heute fast die Hälfte der Mitglieder der deutschen Minderheit in Polen lebt. Interviews mit Einwohnern aus der Woiwodschaft Niederschlesien wurden in das Korpus der deutsch-polnischen Zweisprachigkeit nicht aufgenommen, was damit verbunden ist, dass die Anzahl der deutschstämmigen Bevölkerung dort heute gering ist, auch stammen einige dieser Bewohner aus Oberschlesien (vgl. Gudaszewski 2015, S. 47).

\section{Sprachliche Hintergründe zum Deutschen in Oberschlesien}

Bereits 1965 schreibt Bellmann in der Einleitung seines Schlesischen Sprachatlasses, beim Schlesischen handle es sich um eine nicht mehr bestehende deutsche Sprachlandschaft (vgl. Bellmann 1965, S. 1). Ähnlich urteilt hierüber Wiesinger, der die deutschen Dialekte Schlesiens als einen historischen Gegenstand betrachtet, der lediglich anhand historischen Sprachmaterials zu erforschen wäre (vgl. Wiesinger 1988, S. 645). Die Folgen des Zweiten Weltkriegs: die Grenzverschiebungen und die damit einhergehende Flucht, Vertreibung und Aussiedlung der deutschen Bevölkerung aus den ehemals deutschen Gebieten führten nämlich dazu, dass die Sprecher des Schlesischen, des bis dahin größten zum Ostmitteldeutschen gehörigen deutschen Dialektverbandes, über ganz Deutschland verstreut wurden (vgl. Wiesinger 1983, S. 869). Historisch gesehen stellte die weitläufige schlesische Sprachland- 
schaft keinen einheitlichen Dialekt dar. Vielmehr handelte es sich dabei um einen Verband von mehreren schlesischen Mundarten, die aus dem Ausgleich verschiedener, von Siedlern unterschiedlicher Abstammung mitgebrachter Mundartenelemente entstanden. So schrieb Unwerth: »Der wichtigste Eindruck, den der Beobachter lebender Mundarten in Schlesien empfängt, ist nun der, daß in den einzelnen Gegenden außerordentlich verschieden gesprochen wird.« (Unwerth 1912, S. 100). Jungandreas zufolge entstanden bereits im Mittelalter folgende schlesische Untermundarten: das Lausitzisch-Schlesische, das Neiderländische, das Gebirgsschlesische, das Südschlesische, die oberschlesischen Sprachinseln, das Posensche (vgl. Jungandreas 1937, S. 524). Die Übereinstimmungen zwischen den einzelnen Teilmundarten des Schlesischen erlaubten es, sie zwei Großbereichen zuzuordnen: dem Nord- oder Reichschlesischen und dem Süd- oder Sudetenschlesischen. Dieser Zweiteilung bediente sich bereits Schwarz, der zwischen einem Nord- und einem Südteil des Schlesischen unterschied, und diese Sonderentwicklung der beiden schlesischen Teile auf die politische Lage der Einwanderungszeit zurückführte - den Wettstreit der Piasten um die Kolonisierung im Norden mit den Przemysliden um die im Süden (vgl. Schwarz 1935, S. 20f.). Die einen riefen, Schwarz zufolge, Thüringer und Hessen herbei, die anderen Obermainfranken. Durch eine Binnenwanderung, durch das Vordringen der Obermainfranken von Süden nach Norden und der Thüringer, Rhönfranken und Hessen vom Norden nach Süden entstand jedoch dazwischen keine Sprachscheide, sondern es kam zu einem Ausgleich und zur Herausbildung einer Staffellandschaft (vgl. Schwarz 1935, S. 24f.).

Ähnlich gliederten das Schlesische auch Weinhold, Unwerth und Klapper auf. Weinhold schreibt: »Im Ganzen genügt es, die Mundart des Gebirges und die des Flachlandes [...] zu sondern.« (Weinhold 1862, S. 523). Unwerth und Klapper gebrauchten hierfür die Begriffe Diphthongierungsmundarten, anders Neiderländisch (zur Bezeichnung der Mundart der Niederung) im Norden in Opposition zu der Stammmundart im Süden, der sie das Gebirgsschlesische, das Glätzische und das Lausitzische zuordneten. Als einen Übergang zwischen dem Norden und Süden unterschieden sie die Kräutermundart ${ }^{2}$ in den Kreisen Breslau (Wrocław), Neumarkt (Środa Śląska), Liegnitz (Legnica), Lüben (Lubin), Goldberg-Haynau (ZłotoryjaChojnów), wo sowohl Merkmale der Diphthongierungsmundart als auch der Stammmundart zum Vorschein kamen. Einen Mischcharakter mit südlichen und nördlichen Mundarten wies des Weiteren das Grünbergische auf (vgl. Klapper 1925, S. 56-59; Unwerth 1912, S. 101, 1908, S. 6). Einen Überblick über den Erstreckungsbereich und die Unterteilung der historischen schlesischen Sprachlandschaft liefern die Abb. 1 und 2.

Die deutschsprachigen Äußerungen der Interviewten in Oberschlesien lassen sich keiner dieser dialektalen Varietäten zuordnen. Zwar erwähnen zwei Informantinnen aus Oberglogau (Głogówek) bzw. aus der Nähe von Oberglogau, eine ihrer Groß-

\footnotetext{
2 Als Kräutermundart gilt eine Übergangsmundart vom Gebirgsschlesischen zum Neiderländischen, die im weiten Umkreis von Breslau gesprochen wurde. Die Bezeichnung wird auf die Krautbauern der Breslauer Gegend zurückgeführt, die mehrmals wöchentlich auf dem Breslauer Markt mit ihrem Gemüse handelten. Vgl. Klapper (1925, S. 59) und Menzel (1954, S. 153). Zum Geltungsbereich des schlesischen Dialektverbands und dessen Aufgliederung in Dialektgruppen siehe auch Putschke (1968, S. 138-140).
} 


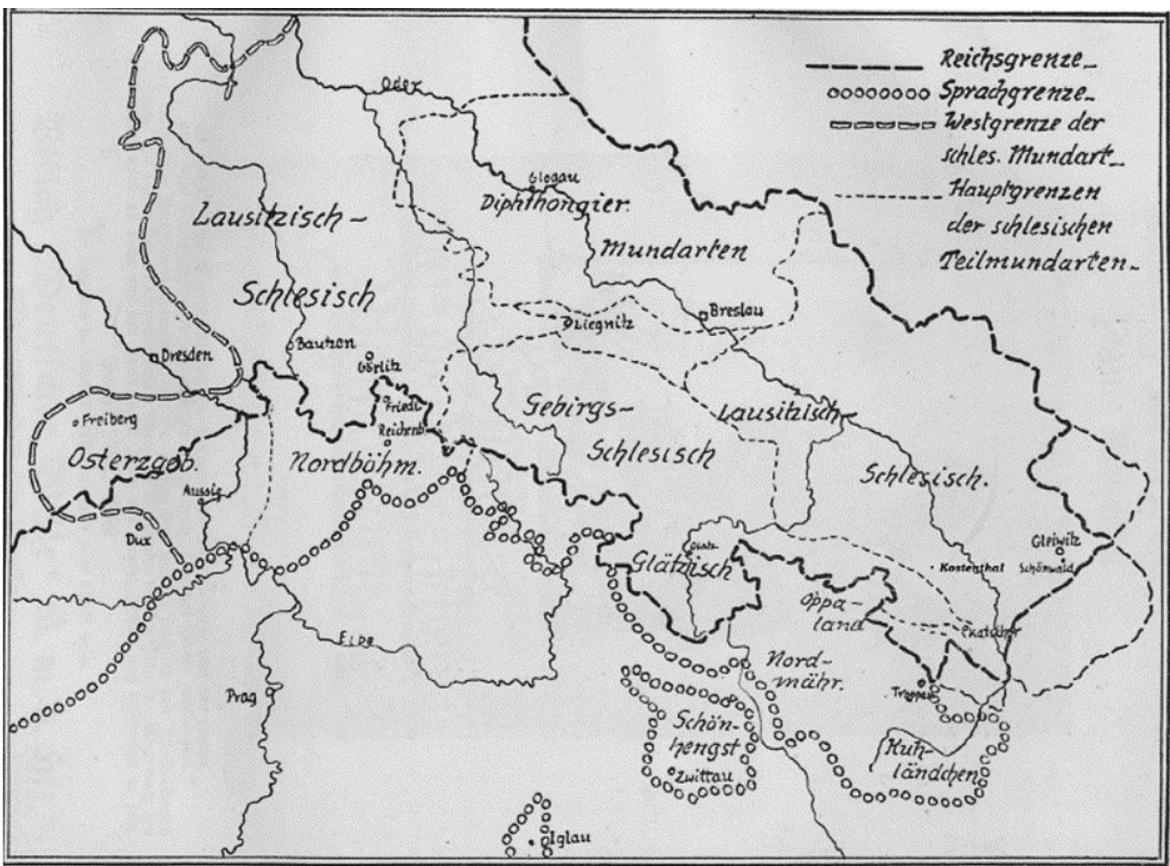

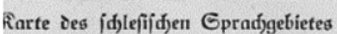

Entworfen von Privatoozent Dr. Gdjwar 3 Prag, gezeidjnet von $\Re$. Syoeller

Abb. 1 Das schlesische Sprachgebiet. (Quelle: Mak (1929, S. 86). Die Karte entspricht der gängigen Gliederung des schlesischen Mundartgebiets nach Wolf von Unwerth (vgl. Unwerth 1908, S. 95))

mütter hätte sich eines deutschen Dialekts bedient, beide geben aber zu, sie hätten ihn nicht gut verstanden und würden selbst Hochdeutsch sprechen. Die Großmutter von IT_GLO stammte aus Kranzdorf (heute Nowy Browiniec), die Großmutter von BI_BRO dagegen aus Deutsch Rasselwitz (heute Racławice Śląskie). Beide Ortschaften gehören historisch zu dem Gebirgsschlesischen.

(1) Richtig von Vater Seite matka byli ( die Mutter war $<$ ) von Rasselwitz. To oni die, oni nie umieli po polsku (>Die, die konnten kein Polnisch $<$ ). Platt, Plattdeutsch barzi ( $>$ mehr $<$ ) Oma, Opa, ni (>nicht $<)$. (BI_BRO)

(2) * Und wissen Sie vielleicht, wie die Großeltern zu Hause gesprochen haben? Plattdeutsch, was ich überhaupt nicht kann, hab auch nicht verstanden. Meine Oma hat Plattdeutsch gesprochen. Sie konnte auch Deutsch.

* Aber die von jetzt von?

Die aus aus Kranzdorf. Die hat, die Oma, die aus Oberglogau, die hat Deutsch gesprochen, aber die Oma aus Kranzdorf hat Plattdeutsch gesprochen. Also da kam ich nicht mit. (IT_GLO)

Auch eine andere Sprecherin bringt ihr Sprachbewusstsein in Bezug auf ihren Deutschgebrauch zum Ausdruck, indem sie das oberschlesische Deutsch als keine dialektale Varietät einstuft. Gleichzeitig ist sie sich jedoch der Eigenart des oberschlesischen Deutsch bewusst: 


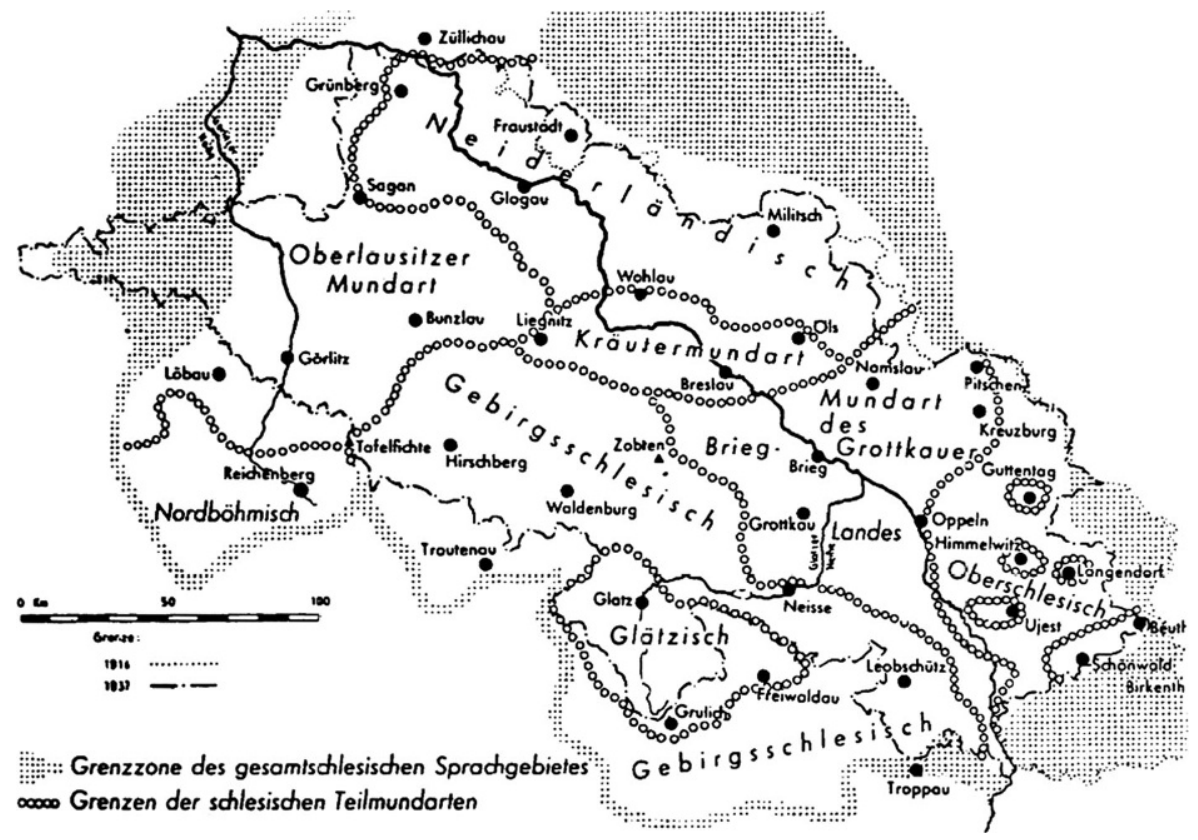

Abb. 2 Die schlesischen Teildialekte. (Quelle: Preuß/Junker 2017, S. 13)

(3) Więc mogę Pani coś powiedzieć, jak ja tutaj, my mówimy tutaj po niemiecku, to każdemu się zdaje, że my ładnie mówimy, jak przyjeżdżamy do Niemiec, to ludzie słyszą, że że że nie jesteśmy tam urodzeni, tak szczerze. Prawda? [...] Moja siostra wyjechała, miała z dwadzieścia dwa lata i to się słyszy. Ona mówi perfekt po niemiecku, moja córka też ma bardzo duży zasób słów, chociaż jest dyslektykiem i to się słyszy, proszę Panią, to to jednak my mamy inny akcent. [...] Ale my mówimy Hochdeutsch, my nie mówimy, na Śląsku nie mówi się jakimś tam, z wyjątkiem małych takich, nie mówimy tak jak w Hamburgu albo w Berlinie ike pike. (CH_RAC)

(>Also ich kann Ihnen etwas sagen, wenn wir hier Deutsch sprechen, scheint es jedem, dass wir schön sprechen, wenn wir nach Deutschland kommen, hören die Leute, dass wir nicht dort geboren sind, um ganz ehrlich zu sein. Nicht wahr? [...] Meine Schwester ist ausgewandert, da war sie so zweiundzwanzig und man kann es hören. Sie spricht perfekt Deutsch, meine Tochter hat auch einen sehr großen Wortschatz, obwohl sie Legasthenikerin ist und man hört es, wissen Sie, wir haben einen anderen Akzent. [...] Aber wir sprechen Hochdeutsch, wir sprechen nicht, in Schlesien sprechen wir nicht so wie in Hamburg oder Berlin ike pike.<)

Auch wenn die Hörer- und Sprecherurteile nicht immer mit den linguistischen Kategorisierungen übereinstimmen müssen, so gilt es festzustellen, dass wegen des Bevölkerungsaustausches als Folge des Zweiten Weltkrieges deutsche Dialekte in Schlesien heute lediglich rudimentär vorkommen. Bei dem meist verbreiteten ober- 
schlesischen Deutsch handelt es sich um eine relativ standardnahe Varietät des Deutschen, dessen Eigenart in hohem Maße auf den Bilingualismus seiner Sprecher zurückgeführt wird (vgl. Pelka 2007, S. 126). Soziolinguistisch und spracherwerbstheoretisch gesehen, lassen sich die im LangGener-Projekt interviewten Sprecher in Oberschlesien in zwei Gruppen einteilen. Die eine bilden elf Interviewte, die angeben, vor 1945 einsprachig Deutsch sozialisiert worden zu sein. Deren standardnahes Deutsch korrespondiert mit einer allgemein verbreiteten Entwicklung, die darauf beruhte, dass die standardsprachliche Überdachung und der Schuleinfluss eine Dialektverschiebung zugunsten des Standarddeutschen nach sich zog (vgl. Wolf 2002, S. 107). Dass geht implizit auch aus den Redeauszügen in den Beispielen 1 und 2 hervor, wo darauf angespielt wird, dass der deutsche Dialekt der Großeltern nicht mehr an die Nachfolgegeneration weitergegeben wurde. Die zweite Gruppe bilden die übrigen neun Informanten aus Oberschlesien, die bereits vor dem Ende des Zweiten Weltkrieges zweisprachig aufgewachsen waren, indem sie neben der offiziellen deutschen Sprache in der Domäne Familie den schlesisch-polnischen Ethnobzw. Regiolekt gebraucht haben. ${ }^{3}$ Deutsch haben sie entweder erst im schulmäßigen Unterricht erworben oder von den Eltern, die sich wiederum die deutsche Sprache oft erst in der Schule angeeignet haben, während die Großeltern eher das Schlesisch-Polnische (den schlesisch-polnischen Ethnolekt) sprachen. Das geht etwa aus den folgenden Redeauszügen hervor, wo die Informanten sich zu ihrem eigenen Sprachgebrauch bzw. dem ihrer Großeltern äußern:

(4) $\mathrm{Zu}$ Hause haben wir nur Schlesisch gesprochen.

* Und woher kannten Sie dann das Deutsche?

No in der Schule, wir gingen dann in die Schule. (KT_DOB)

* Wie sprachen die Großeltern?

Wissen Sie, mehr Polnisch, die haben mehr Polnisch gesprochen. Erst unsere Eltern, die haben Deutsch gesprochen. Und die Großeltern haben so wie jetzt, jetzt sprechen manche Deutsch, manche Polnisch, nicht? (KX_NIE)

Auf der Trias Dialekt-Umgangssprache-Standardsprache wäre das oberschlesische Deutsch heute als eine relativ standardnahe Umgangssprache einzustufen. Auch könnten diesbezüglich die Konzepte Regiolekt, Regionalakzent, regionaler Gebrauchsstandard, Substandard, Nonstandard oder Neuer Substandard wie sie in der einschlägigen Forschungsliteratur diskutiert werden, gebraucht werden (vgl. Ganswindt 2017, S. 40; Księżyk 2008, S. 198). Wie bereits erwähnt, werden die darin vorkommenden Standardabweichungen in der Forschungsliteratur weitgehend auf den mit dem Bilinguismus seiner Sprecher einhergehenden Einfluss des Polnischen bzw. der schlesisch-polnischen Dialekte zurückgeführt (vgl. Księżyk 2008, S. 571). Bei Pelka heißt es:

»Zwar lassen sich nicht alle, doch immerhin viele der darin vorkommenden Abweichungen von der deutschen Standardsprache auf den ständigen Kontakt des Deutschen mit der polnischen oberschlesischen Mundart bzw. der polnischen Standardsprache zurückführen. Somit kann man diese Varietät bzw. deut-

\footnotetext{
${ }^{3} \mathrm{Zu}$ dem Begriff siehe Hentschel (2018, S. 43, 62).
} 
sche Umgangssprache in Oberschlesien als Kontaktvarietät bezeichnen.«(Pelka 2007, S. 126-127)

Damit stimmt auch die Auffassung von Matuschek überein:

»Das Deutsch der Oberschlesier ist eine regionale Variante des Hochdeutschen mit eigenen Entlehnungen aus der polnischen Mundart, besonders in der Lautung.«(Matuschek 1999, S. 195)

Ebenso betrachten Lasatowicz und Weger Sprachkontaktphänomene, die sich in allen Dimensionen des Sprachgebrauchs manifestieren, als wesentliches Merkmal des Oberschlesisch-Deutschen (vgl. Lasatowicz 2002, S. 780; Lasatowicz/Weger 2008, S. 160).

Im Folgenden wird der Fokus nicht auf die sprachkontaktbedingten Eigenheiten des Deutschen in Oberschlesien gerichtet, vielmehr sollen die restdialektalen Merkmale dieser Varietät des Deutschen identifiziert und exemplarisch aufgezeigt werden. $\mathrm{Zu}$ zeigen ist somit, welche Eigenheiten der deutschen Dialekte Schlesiens in der heute in Oberschlesien gesprochenen deutschen Sprache die historischen Dialekte reflektieren. Dabei werden die gesammelten sprachlichen Daten auch im Hinblick darauf analysiert, ob sie von mono- bzw. bilingual aufgewachsenen Sprechern stammen, um gegebenenfalls Unterschiede zwischen ihnen herauszuarbeiten. Anschließend werden die identifizierten dialektalen Merkmale im Hinblick darauf geprüft, ob sie nicht zugleich das sog. landschaftliche Hochdeutsch, die orale Prestigevarietät des ausgehenden 19. Jh., kennzeichnen. Damit könnte der vorliegende Artikel einen kleinen Beitrag zur Behebung des Forschungsdesiderats liefern, das Barbour und Stevenson als $»$ jahrelange Vernachlässigung des umgangssprachlichen Kontinuumsabschnittes« ansehen (Barbour/Stevenson 1998, S. 153).

\section{Remanente dialektale Merkmale im oberschlesischen Deutsch}

Wie bereits erwähnt, lassen sich auch bei den monolingual deutsch aufgewachsenen Sprechern in Oberschlesien die Folgen des Dialektabbaus beobachten, da alle Informanten eine relativ standardnahe regionale deutsche Umgangssprache sprechen. Gewiss dürfte auch der heute leichte Zugang zu den deutschen Medien die Korrektivfunktion der deutschen Standardsprache fördern, sodass in den Aufnahmen lediglich einige rudimentäre Merkmale der deutschen schlesischen Dialekte zu bemerken sind. Insbesondere bei den monolingual aufgewachsenen Sprechern scheinen einige sprachliche Merkmale allerdings noch relativ konsequent und exklusiv vorzukommen.

Während die Entrundung deutscher Vordervokale auch als Einfluss der polnischen Sprache bzw. des schlesisch-polnischen Ethnolekts betrachtet werden könnte (vgl. Pelka 2007, S. 135), so erscheint diese Hypothese im Rahmen des analysierten Korpus nicht unbedingt plausibel. Bei der Analyse des Datenmaterials stellt sich nämlich heraus, dass derartige Entrundungen gerade bei einigen Informanten, die

\footnotetext{
4 Siehe mehr dazu bei Bellmann (1971, S. 10, 16), Rohfleisch (2001, S. 165 f.) und Reiter (1960, S. 64).
} 
behaupteten, vor 1945 einsprachig deutsch gewesen $\mathrm{zu}$ sein, einen fast regulären Charakter haben:

(6) die Zige waren ibefillt (>Die Züge waren überfüllt< KQ_GOG);

(7) kein Leffel (>kein Löffel<KQ_GOG);

(8) die Leute haben jetzt ein bisschen Wohlstand erlebt und da gennen sie sich auch was (>die Leute haben jetzt ein bisschen Wohlstand erlebt und da gönnen sie sich auch was< IT_GLO);

(9) er wird sich kimmern (>er wird sich kümmern< QV_GLO);

(10) wir haben Kerne von den Hihnern gekaut (>wir haben Körner von den Hühnern gekaut< SL_OPO);

(11) die haben das in den Derfern nicht gelernt (>die haben das in den Dörfern nicht gelernt< JP_PRO);

(12) es waren noch zwei Techter (>es waren nich zwei Töchter< IT_GLO);

(13) abe haben wir niemals gehert, dass sie mechte was Polnisch sagen (>aber haben wir niemals gehört, dass sie möchte was Polnisch sagen< BT_KLO).

Bei zweisprachig sozialisierten Informanten kommen ähnliche Phänomene, wenn überhaupt, dann eher vereinzelt vor. Dabei ist es durchaus gerechtfertigt, bei den obigen Beispielen von remanenten dialektalen Merkmalen zu sprechen: Die Entrundung von über zu iber wird auch im Schlesischen Sprachatlas in einigen Regionen verzeichnet (vgl. Bellmann 1967, Karte 34SA). Graebisch zufolge seien die gerundeten Vokale ö und ü dem Schlesischen überhaupt fremd gewesen (vgl. Graebisch 1929, S. 121). Auch Wilhelm Friemel, der weite Teile des oberschlesischen Sprachgebiets in den 30er-Jahren des vergangenen Jahrhunderts dialektgeographisch untersucht hat, betrachtet die Entrundung gerundeter Vokale als eine gemeinschlesische Erscheinung:

»Gemeinschlesisch ist die Entrundung gerundeter Vokale: Fresche, Teppe, Strimpe.« (Friemel 1937, S. 104)

So gesehen ist speziell bei den im Korpus relativ häufig vorkommenden Verbindungen mit kennen + Sprache, etwa Deutsch, Polnisch, Schlesisch kennen, Vorsicht geboten, etwa:

(14) Die kenn Deutsch. (SL_OPO)

(15) Vier Sprachen kennen wir. (FP_MEC)

Zwar liegt bei solchen Realisierungen die Erklärung nahe, dass es sich dabei um eine Musternachbildung (sog. Pattern Replication) aus dem Polnischen znać jezzyk (>eine Sprache kennen $<$ ) handeln dürfte. Aufgrund der Tatsache, dass die Oberschlesier bei dem Verb können den Vordervokal auch in anderen Kontexten entrunden, wie die folgenden Belege veranschaulichen, könnte dies jedoch auch als ein Beispiel für Dialektkontinuität gedeutet werden:

\footnotetext{
5 Siehe dazu auch Bluhme (1964, S. 82). Die Entrundung gerundeter Vokale ist auch eines der Merkmale, die in der hier bereits erwähnten Mundart von Deutsch Rasselwitz besonders auffällt. Vgl. die Mundartprobe von Deutsch Rasselwitz in Księżyk (2008, S. 308-310).
} 
(16) Und da kenn sie nicht so Ski fahrn wie die ganzen Bosses. (JP_PRO)

(17) Man kennte vieles sagen. (LU_KLO)

(18) Heißt, so weit wie wir kennen. (KQ_GOG)

(19) Und da kenn wir nischt machen dagegen. (BT_KLO)

Relativ verbreitet ist auch die Realisierung von $[\varepsilon]$ anstelle des standardgemäßen Reduktionsvokals [e]. Diese Wiedergabe tritt sowohl bei ein- als auch bei zweisprachig sozialisierten Informanten auf:

(20) Obwohl sie die Katzen nicht mag, abe sie kommt sie fittern. (JP_PRO)

(21) Vate (KC_KRA)

(22) Das war doch ein Kriegsverbreche, das war ein Deutsche. (KQ_GOG)

(23) wie ein Bettle (IT_GLO)

(24) Meine Schweste war vier Jahre älte von mir. (KT_DOB)

(25) zwei Söhne und zwei Töchte (LS_OTT)

Auch was diese Realisierung anbelangt, darf nicht vorschnell auf sprachkontaktinduzierte Musterreplikationen geschlossen werden. In den folgenden Belegen handelt es sich beispielsweise nicht um Subjektauslassung in Anlehnung an das Polnische. Wir haben es hier nicht mit einer Flexionsendung des Verbs zu tun, vielmehr wird auch das Personalpronomen er als offenes $[\varepsilon]$ realisiert:

(26) Von wo hat e das Geld gehabt. (QN_BRO)

(27) Deshalb arbeitet e dort. (QN_BRO)

(28) E mechte es kaufen. (JP_PRO)

(29) Hat e zu meinem Vater geschrieben. (BN_PRU)

(30) Da hat e uns mitgenomm. (KQ_GOG)

(31) Die Schuhe, da hat e die Sohle so. (BQ_RAC)

Als remanentes dialektales Merkmal ist die bei einigen Sprechern vereinzelt beibehaltene s-Palatalisierung bei vorausgehendem /r/ zu nennen, zu veranschaulichen etwa mit folgenden Beispielen: ${ }^{6}$

(32) Eine Klosterschwester hat uns zuerscht gelernt. (QV_GLO)

(33) Wo ich das erschte Mal dort hinkam. (IT_GLO)

(34) Wenn du mit dem Wagen gefahren wärscht. (IT_GLO)

(35) Im erschten Weltkrieg (BT_KLO)

(36) Und wenn du wirscht nicht arbeiten, da was? (MS_DOB)

Rudimentären Charakter hat die vereinzelte Erhaltung des unverschobenen $/ \mathrm{p} /$. Gelegentlich wird nämlich die ursprüngliche Geminata $<\mathrm{pp}>$ nicht als die standardgemäße /pf/-Affrikata artikuliert, sondern als der einfache Explosiv [p]: ${ }^{7}$

\footnotetext{
${ }^{6}$ Diese Erscheinung zählt Eberth neben einigen anderen Abweichungen von der Schriftsprache zu den häufigsten »Sprachfehlern« eines Schlesiers, der gewillt ist, Hochdeutsch zu sprechen. Vgl. Eberth (1846, S. 221-226).

7 Die ausgebliebene Verschiebung ist auch bei Unwerth, Jungandreas und Schwarz bei der Beschreibung des Schlesischen belegt (vgl. Unwerth 1912, S. 101; Jungandreas 1937, S. 3; Schwarz 1929, S. 73.).
} 
(37) Da die Frau G* die hat solche solche Teppe mit Suppe gekocht für die Leute. (BT_KLO)

(38) Jemand, der was hier im Koppe hat. (KT_DOB)

(39) Was wir da hatten, einen Topp. (KC_KRA)

Eher beschränkt auf einige Lexeme und gelegentlich bei einigen Sprechern kommt es zu einer Affrikatenvereinfachung von / $/ \mathrm{pf} /$ zur Spirans $/ \mathrm{f} /:^{8}$

(40) Ferde, Ferde, Ferde (KC_KRA)

(41) Und die Mama musste schuften wie ein Ferd, nicht? (IT_GLO)

(42) Das war von der Farrei. (KT_DOB)

(43) Der Farrer lernt hier. (FP_MEC)

Ein vereinzelt, sowohl bei ein- als auch zweisprachig aufgewachsenen Sprechern, vorkommendes dialektales Merkmal ist die stimmhafte Aussprache des intervokalischen f, was Reiter als niederschlesischen Einfluss wertet, etwa (vgl. Reiter 1960, S. 64):

(44) die Bischöwe (LU_KLO)

(45) Ich hab einmal Priwung gehabt (QN_BRO)

(46) Sie hat ja auf Bauernhöwen gearbeitet (IT_GLO)

(47) Da sah er, dass dort Stiewel standen. (KT_DOB)

(48) Die Frau war von Cosel Hawen. (BT_KLO)

Charakteristisch für die sprachbiographischen Interviews in Oberschlesien ist ferner die Elision der Endung -en nach Nasalen. Zwar wird eine weitgehende Assimilation von /en/ nach Nasalkonsonanten, abhängig von Sprechgeschwindigkeit und Stilniveau auch in der Standardsprache festgestellt, im Unterschied zu der Standardsprache geht damit jedoch im oberschlesischen Deutsch nicht unbedingt eine Dehnung des Nasals einher, etwa (vgl. Kohler 1977, S. 219; Meinhold 1973, S. 80 f.; Bluhme 1964, S. 84, 105): ${ }^{9}$

(49) Sie haben sie rausbekomm. (KQ_GOG)

(50) Da hab ich einen kleinen Handwagen genomm. (KT_DOB)

(51) Wo wir von Deutschland kam. (KQ_GOG)

(52) Und meine Eltern sind auch rausgefahren und wohn auch in Wuppertal. (BQ_RAC)

Darüber hinaus treten im Deutsch der interviewten Oberschlesier einige sprachliche Erscheinungen auf, die Lasatowicz als >retrolingual< bezeichnet. Sie entsprechen einem Sprachstand der deutschen Hochsprache von vor 1945, den die Oberschlesier wegen des Jahrzehnte lang fehlenden Kontakts zur deutschen Hochsprache bewahrten (vgl. Lasatowicz 2002, S. 375). Dazu zählt die zuweilen vorkommende

\footnotetext{
8 Zu der variablen Aussprache dieser Affrikate im Anlaut im Schlesischen siehe Schwarz (1929, S. 79).

9 Belege für die en-Apokope nach Nasallauten im Schlesischen finden sich auch bei Schwarz (1929, S. 74). Ebenso sind sie in »Proben der deutschen Mundarten Oberschlesiens« verzeichnet (vgl. Graebisch 1929, S. 122).
} 
Dativmarkierung, die an Substantiven mithilfe der Endung $-e$ vorgenommen wird, etwa: ${ }^{10}$

(53) Bis heute habe ich das noch im Kopfe. (RN_BRO)

(54) mit dem Kinde (KC_KRA)

(55) nach dem Kriege (IT_GLO)

(56) früher im Dorfe (KT_DOB)

(57) von meinem Manne (FP_MEC)

Ebenso ist die Abhebung der prädikativen Verwendung der Zahladjektive vom attributiven Gebrauch durch Schwa-Epithese als ein Anzeichen von regionaler sprachlicher Konservativität zu betrachten, etwa (vgl. Księżyk 2008, S. 210; Langner/Wolf 1996, S. 270): ${ }^{11}$

(58) Dreie sind wir. (SL_OPO)

vs.

(59) In jedem Dorf waren zwei oder drei Schneider. (SL_OPO)

(60) Wir waren zehne. (KC_KRA)

(61) Ich hab gearbeitet vier Jahre oder fümwe. (KQ_GOG)

(62) Da waren zweie aus Hindenburg, zwei Männer. (IT_GLO)

(63) Um dreie kam ich ran. (BQ_RAC)

\section{Dialektmerkmale des Deutschen in Oberschlesien als Kontinuität des landschaftlichen Hochdeutsch}

Das Konzept des landschaftlichen Hochdeutsch stützt sich darauf, dass gegen Ende des 19. Jahrhunderts die Schriftsprache zwar weitgehend vereinheitlicht worden war und als solche auch in den Schulen gelehrt wurde, die Aussprachenormierung sich allerdings erst in der Anfangsphase befand. Vor diesem Hintergrund bildete das landschaftliche Hochdeutsch eine Varietät des Deutschen, die insbesondere von den ausgebildeten Sprechern verwendet wurde, um überregional verstanden zu werden. Angesichts des fehlenden Aussprachestandards dürften sich die Sprecher somit an der Schriftsprache orientieren, wobei deren Sprache weiterhin deutlich regional bzw. dialektal geprägt sein dürfte (vgl. Ganswindt 2017, S. 11-12, 36-37). Von einer solchen Ausspracheorientierung an der Schrift könne zumindest bis zu den 1930ern/1940ern ausgegangen werden, denn erst dann, mit der Rundfunkverbreitung, kamen große Teile der deutschen Bevölkerung mit dem gesprochenen Standard in Kontakt. Mit der Entwicklung des Fernsehens und der Konfrontation der Sprachteilhaber des Deutschen mit der nationalen Aussprachenorm wurden

10 Belegt ist diese sprachliche Erscheinung auch bei Graebisch: »vun dam Zeige [>von dem Zeuge< - F.K.] (Graebisch 1929, S. 125; die Originalschreibweise wird beibehalten), »goob jeedem Kende [>gab jedem Kinde< - F.K.]« (Graebisch 1929, S. 124).

11 In Graebischs »Proben der deutschen Mundarten Oberschlesiens « lassen sich ebenfalls Belege dafür finden: »zwiää Maa(j)dl, sään schun gesturb'm [>zwei Mädels sind schon gestorben< - F.K.]« (Graebisch 1929 , S. 122) vs. »Em a seebne muß der Våter wedder doo sein [>Um siebne muss der Vater wieder da sein<-F.K.]«(Graebisch 1929, S. 125). 
sich die Sprecher der regionalen Begrenzung ihres landschaftlichen Hochdeutsch bewusst, womit die Entstehung der modernen Regionalsprachen angebahnt wurde. Das landschaftliche Hochdeutsch entwickelte sich also zu Regionalsprachen weiter (vgl. Ganswindt 2017, S. 39).

Als eine geeignete Quelle zur Rekonstruktion des landschaftlichen Hochdeutsch erachtet Ganswindt (2017, S. 13, 59) die »Beiträge zur Statistik der Aussprache des Schriftdeutschen « von Wilhelm Viëtor, einem der bekanntesten Aussprachenormierer des Deutschen. Die genannte Publikation stützt sich auf eine Fragebogenuntersuchung, deren Ziel Viëtor folgendermaßen formulierte:

»Gewünscht wird zunächst auskunft über (I) die beim unbefangenen vorlesen gebräuchliche ortsaussprache des schriftdeutschen (LESESPRACHE). Willkommen sind angaben über (II) die mehr oder weniger mundartliche sprache des gebildeten verkehrs, etwa im familienkreise (umgangssprache), und (III) die mundart der niederen klassen (volkssprache). - Werden formen von II und III mitangeführt, so wären solche durch vorgesetztes II, resp. III kenntlich zu machen.«(Viëtor 1888, S. 95)

Die Erhebung der Lesesprache bzw. des landschaftlichen Hochdeutsch im schlesischen Dialektgebiet stützt Viëtor auf einen Fragebogen aus dem Erhebungsort Rawitsch in der damaligen Provinz Posen, wo sich im 17. Jahrhundert Tuchmacher aus Niederschlesien niederließen. ${ }^{12}$ Wie bei allen seinen Informanten handelt es sich auch bei dem Informanten zu Rawitsch, Dr. Alfred Kadler, um keinen Laien in phonetisch-phonologischer Hinsicht.

Innerhalb der lautlichen Merkmale, die in den »Beiträgen zur Statistik der Aussprache des Schriftdeutschen « für Rawitsch verzeichnet wurden, lassen sich fast alle Phänomene auffinden, die im vorliegenden Artikel als Dialektmerkmale in Oberschlesien eingestuft wurden.

1. Die gerundeten palatalen Vokale [y:] [y] werden im landschaftlichen Hochdeutsch in Schlesien ebenfalls entrundet (vgl. Viëtor 1889, S. 249).

2. Die Entrundung von [ë:] [ê] ist bei Viëtor belegt, wobei aus dem Kommentar hervorgeht, dass beim Vorlesen die Schriftsprache Einfluss auf die Aussprache nimmt: »in gewöhnl. Aussprache die schlesischen $e$ [...], beim vorlesen wohl meist $\ddot{o .}$ (Viëtor 1889, S. 248).

Die Tendenz zur Vokalentrundung im landschaftlichen Hochdeutsch Schlesiens bestätigt auch das folgende Zitat Unwerths:

»Was bei gebildeten Schlesiern unter dem Begriff >Schlesische Mundart< geht, sind oft recht verschiedene Dinge. Was jedem bekannt ist, dürfte zunächst die halbmundartliche Aussprache des Schriftdeutschen sein, wie sie in den Städten - innerhalb bestimmter Gesellschaftsklassen auch noch in der Großstadt Breslau - üblich ist. Diese Sprechweise, die gewisse Eigentümlichkeiten der Mund-

12 Vgl. Dziedzictwo językowe Rzeczypospolitej (2021) (http://www.inne-jezyki.amu.edu.pl/Frontend/ Language/Details/30; Stand vom 6.05.2021). 
art (z.B. $i$ für $\ddot{u}$ in >Mühle $<, e$ für $\ddot{o}$ in $>O ̈ l<$ ) bei Benutzung der Schriftsprache verwendet, ist es wohl in erster Linie, die dem Schlesischen den weitverbreiteten Vorwurf der >Häßlichkeit< eingetragen hat.« (Unwerth 1912, S. 99)

3. Die Lenisierung von intervokalischem [f] ist auch in den erfragten Lemmata Briefe, Stiefel, Teufel im landschaftlichen Hochdeutsch erhalten, unter der Anmerkung, dass in III - der Volkssprache - bei Teufel der Verschlusslaut b vorkomme (vgl. Viëtor 1889, S. 251).

4. Die anlautende Deaffrizierung von [pf] ist ebenfalls belegt, auch wenn ebenso Varianten mit der Affrikate verzeichnet werden (vgl. Viëtor 1889, S. 254).

5. Die ausgebliebene Verschiebung von $<\mathrm{pp}>\mathrm{zu}$ [pf] ist als Variante belegt (vgl. Viëtor 1889, S. 254).

6. Ebenso verzeichnet ist die r-Vokalisierung, etwa bei der Endung -er in Feuer, leider oder bitter (vgl. Viëtor 1889, S. 255).

Die übrigen im vorliegenden Artikel behandelten restdialektalen Merkmale des Deutschen in Oberschlesien: die en-Apokope nach Nasallauten, Markierung des Dativs singularischer Substantive mit der Endung - $e$ und die Schwa-Epithese bei Zahladjektiven wurden als koartikulatorische bzw. morphologische Phänomene von dem auf phonetisch-phonologische Variationsphänomene ausgerichteten Fragebogen Viëtors nicht erfasst. Diesbezüglich lassen sich somit keine Schlüsse über ihre Kontinuität im landschaftlichen Hochdeutsch ziehen, wohl aber begegnen sie in der heutigen oberschlesischen deutschen Umgangssprache, wie im Kap. 4 erläutert wurde, zu der sich das landschaftliche Hochdeutsch weiterentwickelt hat.

\section{Fazit}

Gewiss hat der dauerhafte Sprachkontakt, dem das oberschlesische Deutsch unterliegt, Einfluss darauf, dass sich in dieser Varietät des Deutschen eine Reihe von Sprachkontaktphänomenen manifestieren. Die Sprecher selbst sind sich dessen bewusst, dass sie in ihrem Sprachgebrauch aus ihrem gesamten mehrsprachigen Sprachrepertoire schöpfen und die Sprachen, die sie sprechen, sich gegenseitig beeinflussen. Das veranschaulicht die bei den Informanten oft vertretene Auffassung von ihrer »karierten « Sprechweise:

(64) No ja, wir haben schon immer so kariert gesprochen. Halb Deutsch, halb Polnisch. (FP_MEC)

Dabei erfährt das deutsche partizipiale Adjektiv nach dem Muster des Polnischen eine Polysemiekopierung. Es wird nämlich nicht im Sinne von >unlogisch, durcheinander verwendet, sondern in der Bedeutung des polnischen Äquivalents »w kratkę«, d.h. >abwechselnd<.

Auch die zunächst monolingual aufgewachsenen Sprecher bekunden mehrfach ihre Zugehörigkeit zu der zweisprachigen Sprachgemeinschaft und spielen dabei auf die sprachkontaktinduzierten Eigentümlichkeiten des oberschlesischen Deutsch an. 
So verweist in dem folgenden Redeausschnitt der Sohn der Informantin darauf, dass die von ihr verwendete Pilzbezeichnung nicht standarddeutsch sei. Die Sprecherin scheint skeptisch bzw. desinteressiert darauf zu reagieren:

(65) No heut zum Beispiel miałach (>hatte ich $<$ ) eine Pilzensuppe, aber von zwei zweierlei Pilzen, bo (>denn $<$ ) die Hihnerpilze die Kurkis znoł Pani ( Pfifferlinge, kennen Sie die $<$ ? (KQ_GOG)

* Ja.

No das die sind gut für die Suppe nicht. No da kaufe immer, wenn sie im Geschäft sind kurki ( $>$ Pfifferlinge $<$ ) und die die Hihnerpilze und dann jeszcze (>noch $<$ ) Cham*

Wie heißen die Hihnerpilze? Wie heißen sie richtig? (LQ_GOG)

No Hihnerpilze. (KQ_GOG)

* Pfifferlinge.

Pfefferlinge, Pfefferlinge. (LQ_GOG)

Pfefferlinge? Alles möglich, das ist so. Das ist Wurst, egal. (KQ_GOG)

Dabei stellt sich heraus, dass es sich bei der Bezeichnung Hühnerpilze keineswegs um eine Ad-hoc-Lehnübertagung des polnischen Begriffs kurki handelt. Die Bezeichnung Hihnerpilze wurde beispielsweise auch in den Belegorten Deutsch Rasselwitz (heute Racławice Śląskie) und Dobrau (heute Dobra) verzeichnet, in Ortschaften also, die nur zehn bis 30 Kilometer von dem Wohnort der Informantin entfernt liegen (vgl. Bellmann 1965, S. 7). Auch im Schlesischen Wörterbuch von Mitzka wird der Begriff Hühnerpilze alternativ zu Pfifferlingen angeführt (vgl. Mitzka 1963, S. 570).

Ähnlich darf auch bei anderen sprachlichen Erscheinungen, die von der standarddeutschen Norm abweichen, nicht vorschnell auf eine Sprachkontaktinduzierung geschlossen werden. Wie bereits gezeigt wurde, hat der Sprachkontakteinfluss nicht immer das alleinige und größte Erklärungspotenzial für manche im oberschlesischen Deutsch der interviewten Informanten auftretende Phänomene. Zwar ist das heutige Deutsch in Oberschlesien in der Tat standardnah, ${ }^{13}$ was neben dem Einfluss der Aussprache in den Massenmedien sehr stark auch damit korrespondiert, dass das ostmitteldeutsche Dialektgebiet, zu dem das Schlesische gehört, die deutsche Einheitssprache historisch stark geprägt hatte, weswegen es heute generell dem Standard nahe steht (vgl. Barbour/Stevenson 1998, S. 152), dennoch lassen sich einige im oberschlesischen Deutsch vorkommende sprachliche Erscheinungen als Kontinuität der historischen deutschen Dialekte Schlesiens aufzeigen. Die identifizierten remanenten Dialektmerkmale des Deutschen, die auch heute in der oberschlesischen Umgangssprache realisiert werden, erwiesen sich, soweit das mithilfe der »Beiträge zur Statistik der Aussprache des Schriftdeutschen« rekonstruiert werden konnte, ebenfalls als Merkmale des sog. landschaftlichen Hochdeutsch, die die Schlesier auch bei der Aussprache des Schriftdeutschen realisiert haben. Bei diesen abbauresistenten Merkmalen, die sowohl ins landschaftliche Hochdeutsch, die historische Vorstufe der hier beschriebenen Umgangssprache, übernommen wurden, als auch

13 Bereits Bluhme (1964) ging von einem schlesisch gefärbten Hochdeutsch aus, dies allerdings im oberschlesischen Industriegebiet. 
in die heutige regionale Umgangssprache Eingang fanden, handelt es sich jedoch um keine kleinräumigen Phänomene, die nur für einzelne schlesische Teilmundarten charakteristisch waren, vielmehr sind es Merkmale, die weitläufiger, etwa im ganzen mitteldeutschen Raum verbreitet sind. Die von den interviewten Oberschlesiern bewahrten Merkmale der schlesischen deutschen Dialekte stellen allerdings lediglich einige restdialektale Phänomene dar, zudem ist die Varietät, in der sie vorkommen, im Rückgang begriffen. Zwar wird den Ergebnissen des letzten Zensus von 2011 zufolge die deutsche Sprache weiterhin als Familiensprache von ca. 96 Tsd. Menschen in Polen verwendet, die Tendenz ist allerdings fallend: Immerhin haben sich dazu 2002 über 200 Tsd. Menschen bekannt (vgl. Gudaszewski 2015, S. 72). Obwohl nach der Grenzverschiebung 1945 zunächst noch viele deutschstämmige Einwohner in den ehemals deutschen Gebieten, die Polen einverleibt wurden, geblieben sind, so ist dieser Anteil nach und nach infolge der Auswanderungswellen geschrumpft. Was den Deutschgebrauch angeht, so hat das Streben Nachkriegspolens nach einem mononationalen Staat, damit einhergehende Repressalien, die die einheimische Bevölkerung für die Verwendung der deutschen Sprache auch im privaten Bereich erlitten hatte, dazu geführt, dass es nach und nach zu einem Bruch bei der Tradierung der deutschen Sprache an die nachfolgenden Generationen kam. Die hier beschriebene Varietät des Deutschen mit ihren remanenten Dialektmerkmalen ist somit vom Aussterben bedroht.

\section{Liste der zitierten Informant_innen}

- BI_BRO, eine Frau, wohnhaft in Nowy Browiniec, geboren 1931 in Deutsch Probnitz

- BN_PRU, eine Frau, wohnhaft in Biała Prudnicka, geboren 1931 in Körnitz

- BQ_RAC, eine Frau, wohnhaft in Racibórz, geboren 1941 in Falkendorf

- BT_KLO, ein Mann, wohnhaft in Kędzierzyn-Koźle, geboren 1935 in Klodnitz

- CH_RAC, eine Frau, wohnhaft in Racibórz, geboren 1940 in Hultschin

- FP_MEC, eine Frau, wohnhaft in Mechnica, geboren 1929 in Mechnitz

- IT_GLO, eine Frau, wohnhaft in Głogówek, geboren 1938 in Oberglogau

- JP_PRO, eine Frau, wohnhaft in Prószków, geboren 1926 in Proskau

- KC_KRA, ein Mann, wohnhaft in Krapkowice, geboren 1929 in Neustadt

- KQ_GOG, eine Frau, wohnhaft in Gogolin, geboren 1933 in Gogolin

- KT_DOB, ein Mann, wohnhaft in Dobrzeń Wielki, geboren 1929 in Groß Döbern

- KX_NIE, eine Frau, wohnhaft in Nieznaszyn, geboren 1930 in Niesnaschin

- LQ_GOG, ein Mann, wohnhaft in Gogolin, geboren 1956 in Gogolin

- LS_OTT, ein Mann, wohnhaft in Otmice, geboren 1930 in Ottmütz

- LU_KLO, ein Mann, wohnhaft in Kędzierzyn-Koźle, geboren 1940 in Klodnitz

- MS_DOB, eine Frau, wohnhaft in Dobrzeń Wielki, geboren 1929 in Groß Döbern

- QV_GLO, ein Mann, wohnhaft in Głogówek, geboren 1936 in Oberglogau

- QN_BRO, ein Mann, wohnhaft in Brożec, geboren 1942 in Beuthen

- SL_OPO, eine Frau, wohnhaft in Opole, geboren 1939 in Oppeln

Funding Open access funding provided by University of Opole. 
Open Access Dieser Artikel wird unter der Creative Commons Namensnennung 4.0 International Lizenz veröffentlicht, welche die Nutzung, Vervielfältigung, Bearbeitung, Verbreitung und Wiedergabe in jeglichem Medium und Format erlaubt, sofern Sie den/die ursprünglichen Autor(en) und die Quelle ordnungsgemäß nennen, einen Link zur Creative Commons Lizenz beifügen und angeben, ob Änderungen vorgenommen wurden.

Die in diesem Artikel enthaltenen Bilder und sonstiges Drittmaterial unterliegen ebenfalls der genannten Creative Commons Lizenz, sofern sich aus der Abbildungslegende nichts anderes ergibt. Sofern das betreffende Material nicht unter der genannten Creative Commons Lizenz steht und die betreffende Handlung nicht nach gesetzlichen Vorschriften erlaubt ist, ist für die oben aufgeführten Weiterverwendungen des Materials die Einwilligung des jeweiligen Rechteinhabers einzuholen.

Weitere Details zur Lizenz entnehmen Sie bitte der Lizenzinformation auf http://creativecommons.org/ licenses/by/4.0/deed.de.

\section{Literatur}

Barbour, Stephen/Stevenson, Patrick (1998): Variation im Deutschen. Soziolinguistische Perspektiven. Berlin/New York: de Gruyter.

Bellmann, Günter (1965): Schlesischer Sprachatlas. Bd. 2 Wortatlas. Marburg: Elwert.

Bellmann, Günter (1967): Schlesischer Sprachatlas. Bd. 1 Laut- und Formenatlas. Marburg: Elwert.

Bellmann, Günter (1971): Slavoteutonica. Lexikalische Untersuchungen zum slawisch-deutschen Sprachkontakt im Ostmitteldeutschen. Berlin/New York: de Gruyter.

Bluhme, Hermann (1964): Beitrag zur deutschen und polnischen Mundart im oberschlesischen Industriegebiet. Unter besonderer Berücksichtigung phonometrischer Methoden. Den Haag: Nijhoff.

Dziedzictwo językowe Rzeczpospolitej (2021) http://www.inne-jezyki.amu.edu.pl/Frontend/Language/ Details/30

Eberth, Karl (1846): Ueber die gewöhnlichsten Sprachfehler der Schlesier. In: Schlesische Provinzialblätter, Bd. 123, S. 217-226.

Friemel, Wilhelm (1937): Die Mundart von Oberglogau. In: Schlesische Geschichtsblätter 3, S. 104-108.

Ganswindt, Brigitte (2017): Landschaftliches Hochdeutsch. Rekonstruktion der oralen Prestigevarietät im ausgehenden 19. Jahrhundert. Stuttgart: Franz Steiner Verlag.

Graebisch, Friedrich (1929): Proben der deutschen Mundarten Oberschlesiens. In: Der Oberschlesier, Jahrgang 11, Heft 2, S. 120-128.

Gudaszewski, Grzegorz (2015): Struktura narodowościowo-etniczna, jezykowa i wyznaniowa ludności Polski. Narodowy Spis Powszechny Ludności i Mieszkań 2011. Warszawa: GUS. In: https:// stat.gov.pl/spisy-powszechne/nsp-2011/nsp-2011-wyniki/struktura-narodowo-etniczna-jezykowa-iwyznaniowa-ludnosci-polski-nsp-2011,22,1.html (29.01.2021).

Heine, Bernd/Kuteva, Tania (2005): Language Contact and Grammatical Change. Cambridge: Cambridge Univ. Press.

Hentschel, Gerd (2018): Śląski: gwara - dialekt - język? Spojrzenie z zewnątrz. In: Jolanta Tambor (Hg.): Pogranicza, mniejszości, regiony. Etnolingwistyka. Katowice: Wydawnictwo Uniwersytetu Śląskiego, S. 41-66.

Jungandreas, Wolfgang (1937): Zur Geschichte der schlesischen Mundart im Mittelalter. Breslau: Maruschke \& Berendt.

Klapper, Joseph (1925): Schlesische Volkskunde auf kulturgeschichtlicher Grundlage. Breslau: Ferdinand Hirt.

Kohler, Klaus J. (1977): Einführung in die Phonetik des Deutschen. Berlin: Erich Schmidt Verlag.

Księżyk, Felicja (2008): Die deutsche Sprachinsel Kostenthal. Geschichte und Gegenwart. Berlin: trafo Verlag.

Langner, Helmut/Wolf, Norbert Richard ( $\left.{ }^{7} 1996\right)$ (Hg.): Wilhelm Schmidt. Geschichte der deutschen Sprache. Stuttgart/Leipzig: S. Hirzel.

Lasatowicz, Maria Katarzyna (2002): Zur Dynamik sprachlicher Wirklichkeiten im Raum Oberschlesien. In: Edward Białek/Manfred Durzak/Marek Zybura (Hg.): Literatur im Zeugenstand: Beiträge zur deutschsprachigen Literatur- und Kulturgeschichte. Festschrift zum 65. Geburtstag von Hubert Orłowski. Frankfurt a. M.: Peter Lang, S. 773-788. 
Lasatowicz, Maria Katarzyna/Weger, Tobias (2008): Polen. In: Ludwig M. Eichinger/Albrecht Plewnia/ Claudia M. Riehl (Hg.): Handbuch der deutschen Sprachminderheiten in Mittel- und Osteuropa. Tübingen: Narr, S. 145-169.

Mak, Wilhelm (1929): Die schlesischen Mundarten: Deutsche Mundarten in Oberschlesien. In: Der Oberschlesier 11/2, S. 1-86.

Matuschek, Herbert (1998/1999): Das Polnisch der Oberschlesier. Zu den Kontroversen um ein Idiom (Fortsetzung). In: Oberschlesisches Jahrbuch. Band 14/15, S. 193-213.

Meinhold, Gottfried (1973): Deutsche Standardaussprache. Lautschwächungen und Formstufen. Jena: Friedrich-Schiller-Univ.

Menzel, Wilhelm (1954): Die schlesische Mundart. In: Karl Hausdorff (Hg.): Unser Schlesien. Stuttgart: Mayer, S. 142-154.

Mitzka, Walther (1963): Schlesisches Wörterbuch. Bd. 1. A-H. Berlin: de Gruyter.

Pelka, Daniela (2007): Methode und Ergebnisse einer kontaktlinguistischen Untersuchung in Oberschlesien. In: Germanoslavica. Zeitschrift für germano-slawische Studien 18/1-2, S. 125-146.

Preuß, Friedrich Wilhelm/Junker, Ullrich (2017): Woas die Stoare pfeifa. Festschrift 35 Jahre erfolgreiche Arbeit Arbeitskreis für schlesische Mundart. Wangen. In: http://jbc.jelenia-gora.pl/Content/26863/ JEL_28820_2017_Festschrift---35-Jah_woas_band_21.pdf (25.01.2021).

Putschke, Wolfgang (1968): Ostmitteldeutsche Dialektologie. In: Ludwig Erich Schmidt (Hg.): Germanische Dialektologie. Festschrift für Walther Mitzka zum 80. Geburtstag. Wiesbaden: Steiner, S. 105-154. (=Zeitschrift für Mundartforschung; Beihefte. Neue Folge; 5).

Reiter, Norbert (1960): Die polnisch-deutschen Sprachbeziehungen in Oberschlesien. Wiesbaden: Harrassowitz.

Rohfleisch, Irene (2001): Sprachsituation und Sprachverhalten in Teilen des heutigen Oberschlesiens. Berlin: Dissertation.de.

Schwarz, Ernst (1935): Sudetendeutsche Sprachräume. München: Reinhardt.

Schwarz, Ernst (1929): Die schlesische Mundart. In: Der Oberschlesier, Jahrgang 11, Heft 2, S. 71-83.

Unwerth, Wolfgang von (1908): Die schlesische Mundart in ihren Lautverhältnissen grammatisch und geographisch dargestellt. Breslau: M. \& H. Marcus.

Unwerth, Wolfgang von (1912): Die schlesische Mundart. In: Robert Henseling (Hg.): Schlesisches Jahrbuch 1913. Berlin: Dietrich Reimer, S. 99-104.

Viëtor, Wilhelm (1888): Beiträge zur Statistik der Aussprache des Schriftdeutschen I. In: Phonetische Studien 1, S. 95-114.

Viëtor, Wilhelm (1889): Beiträge zur Statistik der Aussprache des Schriftdeutschen III. In: Phonetische Studien 2, S. 243-258.

Weinhold, Karl (1862): Schlesien in sprachlicher Hinsicht. In: Schlesische Provinzialblätter 1, S. 521-524.

Wiesinger, Peter (1983): Die Einteilung der deutschen Dialekte. In: Werner Besch/Ulrich Knoop/Wolfgang Putschke/Herbert Ernst Wiegand (Hg.): Dialektologie. Ein Handbuch zur deutschen und allgemeinen Dialektforschung. Bd. 2. Berlin/New York: de Gruyter, S. 801-900.

Wiesinger, Peter (1988): Die Vokalentwicklungen des Neiderländisch-Schlesischen in strukturgeographisch-strukturhistorischer Sicht. Eine Studie zur historischen Dialektologie. In: Jacek Fisiak (Hg.): Historical Dialectology - Regional and Social. Berlin u.a.: Mouton de Gruyter, S. 645-678 (=Trends in Linguistics. Studies and Monographs. 37).

Wolf, Norbert Richard (2002): Regionen entstehen, indem die Leute miteinander reden. Oder: Regionalisierungsprozesse in rezenten Dialekten. In: Regionalität als Kategorie der Sprach- und Literaturwissenschaft. Hg. vom Instytut Filologii Germańskiej der Uniwersytet Opolski. Frankfurt (Main) u.a.: Peter Lang, S. 97-110 (=Oppelner Beiträge zur Germanistik 6). 